PROCEEDINGS OF THE

AMERICAN MATHEMATICAL SOCIETY

Volume 129, Number 8, Pages 2235-2238

S 0002-9939(01)05848-8

Article electronically published on January 23, 2001

\title{
HYPERCENTRAL UNITS IN INTEGRAL GROUP RINGS
}

\author{
YUANLIN LI AND M. M. PARMENTER
}

(Communicated by Steven D. Smith)

\begin{abstract}
In this note, we show that when $G$ is a torsion group the second center of the group of units $U(\mathbb{Z} G)$ of the integral group ring $\mathbb{Z} G$ is generated by its torsion subgroup and by the center of $U(\mathbb{Z} G)$. This extends a result of Arora and Passi (1993) from finite groups to torsion groups, and completes the characterization of hypercentral units in $\mathbb{Z} G$ when $G$ is a torsion group.
\end{abstract}

Let $\mathbb{Z} G$ denote the integral group ring of a torsion group $G, U(\mathbb{Z} G)$ the group of units of such a group ring and $V(\mathbb{Z} G)$ the subgroup of units of augmentation 1 .

In the case where $G$ is finite, the ascending central series $Z_{n}(V(\mathbb{Z} G))$ was studied in [1] and 2. In [1] it was shown that $Z_{n+1}(V(\mathbb{Z} G))=Z_{n}(V(\mathbb{Z} G))$ whenever $n \geq 2$, while the main result of [2] states that $Z_{2}(V(\mathbb{Z} G))=T \cdot Z_{1}(V(\mathbb{Z} G))$ where $T$ denotes the torsion subgroup of $Z_{2}(V(\mathbb{Z} G))$. The first of these results was extended to torsion groups in [5], and the question of whether or not the second result could be similary extended was Open Problem 5 in 8$]$.

In this note, we show that the second result can indeed be extended to torsion groups. Although Blackburn's theorem [3] on the intersection of nonnormal subgroups of finite groups played a significant role in the investigation of the structure of the second center in 2], this result cannot be applied directly to our case. Instead, our argument focusses on the importance of Bass cyclic, bicyclic and Hoechsmann units in integral group rings.

Notation and terminology will follow that in [10.

The following lemma is crucial to our approach. Although the argument given can be found in [5] (as part of the proof of Theorem 2), we include it here for completeness.

Lemma 1. If $G$ is a torsion group, $\left[Z_{2}(V(\mathbb{Z} G))\right]^{2} \subseteq Z_{1}(V(\mathbb{Z} G))$.

Proof. If $v \in Z_{2}(V(\mathbb{Z} G))$ and $g \in G$, then $c=v g v^{-1} g^{-1}$ is in $Z_{1}(V(\mathbb{Z} G))$. Since $c g$ is of finite order, it follows that $c$ is also of finite order and hence is in $G$ [9, p.46]. Thus $v$ is in $N_{V(\mathbb{Z} G)}(G)$ and so $v^{2} \in G \cdot Z_{1}(V(\mathbb{Z} G))$ [10, p.32]. It follows that if $\left[Z_{2}(V(\mathbb{Z} G))\right]^{2}$ is not contained in $Z_{1}(V(\mathbb{Z} G))$, then there must exist a group element $h$ in $Z_{2}(V(\mathbb{Z} G)) \backslash Z_{1}(V(\mathbb{Z} G))$. But then if $u$ is any unit in $\mathbb{Z} G,[u, h]=h_{0} \in Z_{1}(G)$ and there exists a positive integer $n=n(u)$ such that $u^{n} h u^{-n}=h$. It follows from Theorem 1.2 of [7] that the exponent of $Z_{1}(G)$ is 2 , and so for all $u_{2} \in Z_{2}(V(\mathbb{Z} G))$

Received by the editors August 3, 1999 and, in revised form, December 24, 1999.

2000 Mathematics Subject Classification. Primary 16S34, 20 C07.

This research was supported in part by grants from the Natural Sciences and Engineering Research Council. 
and all $g \in G$, we have $\left[u_{2}^{2}, g\right]=\left[u_{2}, g\right]^{2}=1$. This contradiction finishes the proof.

We remark that the relationship $Z_{2}(V(\mathbb{Z} G)) \subseteq N_{V(\mathbb{Z} G)}(G)$, obtained in the above proof, was also noted in [6] and, as a consequence, some partial results on the problem of when $Z_{2}(V(\mathbb{Z} G))=T \cdot Z_{1}(V(\mathbb{Z} G))$ were obtained in that paper.

Recall that a torsion group $G$ is said to be a $Q^{*}$-group if $G$ has an Abelian normal subgroup $A$ of index 2 which has an element $a$ of order 4 such that for all $h \in A$ and all $g \in G \backslash A, g^{2}=a^{2}$ and $g^{-1} h g=h^{-1}$.

Here is our main result.

Theorem 2. Let $G$ be a torsion group. Then $Z_{2}(V(\mathbb{Z} G)) \neq Z_{1}(V(\mathbb{Z} G))$ if and only if $G$ is a $Q^{*}$-group. In that case, either $G$ is a Hamiltonian 2-group and $Z_{2}(V(\mathbb{Z} G))=G$ or $Z_{2}(V(\mathbb{Z} G))=\langle a\rangle Z_{1}(V(\mathbb{Z} G))$ where a is any element of the type defined above.

Proof. First we recall that if $G$ is a $Q^{*}$-group and $a$ is as defined above, then $\langle a\rangle$ is normal in $V(\mathbb{Z} G)$ (see [4] or the last paragraph on p.5505 of [7]). So if $u$ is any unit in $V(\mathbb{Z} G)$, either $a u=u a$ or $a u=a^{2}(u a)$. Since $a^{2}$ is central, this shows that $a \in Z_{2}(V(\mathbb{Z} G)) \backslash Z_{1}(V(\mathbb{Z} G))$, and we have also established that $\langle a\rangle Z_{1}(V(\mathbb{Z} G)) \subseteq Z_{2}(V(\mathbb{Z} G))$ in this case.

Now let $G$ be a torsion group such that $Z_{2}(V(\mathbb{Z} G)) \neq Z_{1}(V(\mathbb{Z} G))$ and choose $z_{2} \in Z_{2}(V(\mathbb{Z} G)) \backslash Z_{1}(V(\mathbb{Z} G))$. Lemma 1 tells us that $z_{2}^{2} \in Z_{1}(V(\mathbb{Z} G))$ and hence $\left[z_{2}, u\right]$ is a central unit of order $\leq 2$ in $\mathbb{Z} G$ (and so lies in $G$ ) for all $u \in V(\mathbb{Z} G$ ).

We will show that $G$ is a $Q^{*}$-group and that the required condition is satisfied.

First assume that $G$ is not a Dedekind group. If $x, y \in G$ satisfy $y x y^{-1} \notin\langle x\rangle$, consider the nontrivial bicyclic unit

$$
u=1+\hat{x} y(1-x)
$$

where $\hat{x}=1+x+x^{2}+\cdots+x^{o(x)-1}$. We know that $z_{2} u=u z_{2} c$ for some $c \in Z_{1}(G)$ of order $\leq 2$. It follows that $z_{2} u^{2}=u^{2} z_{2}$. Since $u^{2}=1+2 \hat{x} y(1-x)$, this means that $z_{2} u=u z_{2}$. Thus $z_{2}(\hat{x} y(1-x)) z_{2}^{-1}=\hat{x} y(1-x)$ and, since $z_{2} \in N_{V(\mathbb{Z} G)}(G)$ (as seen above), we conclude that $z_{2} y z_{2}^{-1}=x^{i} y$ for some $i$. Now assume $w \in G$ satisfies $w x w^{-1} \in\langle x\rangle$. Replacing $y$ by $y w$ in the above argument, we obtain $z_{2} y w z_{2}^{-1}=x^{j} y w$ for some $j$. Recalling that $x^{i}$ and $x^{j}$ are central, this gives $z_{2} w z_{2}^{-1}=\left(x^{i} y\right)^{-1}\left(x^{j} y w\right)=x^{j-i} w$.

We have proved that if $\langle x\rangle$ is a nonnormal subgroup of $G$, then $\left[z_{2}, g\right] \in\langle x\rangle$ for all $g \in G$ (note that this means $\left[z_{2}, g\right]$ can take on only two distinct values as $g$ ranges through $G$ ). In particular, this says that $\left[z_{2}, x\right] \in\langle x\rangle$ whenever $\langle x\rangle$ is not normal in $G$. But if $\langle x\rangle$ is normal in $G$, it is still the case that $\left[z_{2}, x\right] \in\langle x\rangle$ (since the conjugacy class sum of $x$ is central and $z_{2}$ is in $N_{V(\mathbb{Z} G)}(G), z_{2} x z_{2}^{-1}$ must be a conjugate of $x$ in $G)$. Hence $\left[z_{2}, g\right] \in\langle g\rangle$ holds for all $g \in G$.

Since $\left[z_{2}, g\right]$ is central of order $\leq 2$, it follows that $\left[z_{2}, g\right]=1$ whenever $g \in G$ is of odd order. We now show that $\left[z_{2}, g\right] \neq 1$ can only occur when $g$ is of order 4 .

First note that if $g \in G$ is of order 2 , then $\left[z_{2}, g\right]=1$ or $g$ by above. But $\left[z_{2}, g\right]=g$ implies $z_{2} g z_{2}^{-1}=1$ which is impossible, so we conclude that $\left[z_{2}, g\right]=1$.

Next assume that $g \in G$ is of order $2^{m}$ where $m \geq 3$ and consider the Bass cyclic unit (see [10] for details)

$$
u=\left(1+g+g^{2}\right)^{2^{m-1}}+\frac{1-3^{2^{m-1}}}{2^{m}} \hat{g} .
$$


We know that $z_{2} g z_{2}^{-1}=g$ or $z_{2} g z_{2}^{-1}=g^{2^{m-1}+1}$. Say the latter holds. We also know that $z_{2} u z_{2}^{-1}=u c$ where $c$ is a central unit of order $\leq 2$, and it is clear that $c=1$ or $c=g^{2^{m-1}}$. If $c=1$, we obtain $\left(1+g^{2^{m-1}+1}+g^{2}\right)^{2^{m-1}}=\left(1+g+g^{2}\right)^{2^{m-1}}$ which is not true because the coefficient of $g$ on the right-hand side is $2^{m-1}$ while the coefficient of $g$ on the left-hand side is greater than $2^{m-1}$. If $c=g^{2^{m-1}}$, we obtain $\left(1+g^{2^{m-1}+1}+g^{2}\right)^{2^{m-1}}=\left(\left(1+g+g^{2}\right) g\right)^{2^{m-1}}$ which is also not true because the coefficients of $g^{2^{m-1}}$ are different on the two sides. So the case where $z_{2} g z_{2}^{-1}=g^{2^{m-1}+1}$ is impossible and we must have $\left[z_{2}, g\right]=1$.

Finally assume that $g \in G$ is of order $2^{m} s$ where $m \geq 1, s$ is odd and $s>1$. We know that $z_{2} g=g z_{2} c$ where $c$ is central of order $\leq 2$. Since $z_{2} g^{s}=g^{s} z_{2} c$ and $g^{s}$ is of order $2^{m}$, it follows from the last two paragraphs that we may assume $m=2$. Also, if $p$ is an odd prime such that $p \mid s$, then $z_{2} g^{\frac{s}{p}}=g^{\frac{s}{p}} z_{2} c$. So to complete this part of the proof, we may assume that $g$ is of order $4 p$. Say $z_{2} g z_{2}^{-1}=g^{2 p+1}$. If $p>3$, consider the Bass cyclic unit

$$
u=\left(1+g+g^{2}\right)^{2(p-1)}+\frac{1-3^{2(p-1)}}{4 p} \hat{g} .
$$

If $z_{2} u z_{2}^{-1}=u$, we obtain $\left(1+g^{2 p+1}+g^{2}\right)^{2(p-1)}=\left(1+g+g^{2}\right)^{2(p-1)}$ which is impossible because the coefficients of $g^{4 p-1}$ are different on the two sides. If $z_{2} u z_{2}^{-1}=u g^{2 p}$, we get $\left(1+g^{2 p+1}+g^{2}\right)^{2(p-1)}=\left(1+g+g^{2}\right)^{2(p-1)} g^{2 p}$, which is also impossible because the coefficients of $g^{2 p}$ are different. We have a contradiction, and must conclude that $\left[z_{2}, g\right]=1$. If $p=3$, consider the Hoechsmann unit (see [10] for details)

$$
v=\left(1+g^{5}+g^{10}+g^{3}+g^{8}\right)^{2}-2 \hat{g} .
$$

As before, both $z_{2} v z_{2}^{-1}=v$ and $z_{2} v z_{2}^{-1}=v g^{6}$ would contradict $z_{2} g z_{2}^{-1}=g^{7}$. We again conclude that $\left[z_{2}, g\right]=1$.

Now let $H=\left\{x \in G \mid z_{2} x=x z_{2}\right\}$. It is easy to see that $H \triangleleft G$. If $x \in G \backslash H$ and $y \in G \backslash H$, we know that $x$ and $y$ are both of order 4 and hence that $z_{2} x=$ $x^{3} z_{2}, z_{2} y=y^{3} z_{2}$ where $x^{2}=y^{2}$ (as noted earlier) is central. Hence $z_{2} x y=x^{3} y^{3} z_{2}=$ $x y z_{2}$. We conclude that $|G / H|=2$.

If $h \in H$ and $x \in G \backslash H$, then $x h \in G \backslash H$. It follows that $x$ and $x h$ are both of order 4 and $(x h)^{2}=x^{2}$, giving $x h x^{-1}=h^{-1}$. If $k \in H$ also, we have $x h k x^{-1}=$ $(h k)^{-1}$ while $x h k x^{-1}=\left(x h x^{-1}\right)\left(x k x^{-1}\right)=h^{-1} k^{-1}$, giving $h k=k h$. Hence $H$ is Abelian.

Finally, note that when $\left[z_{2}, g\right]=c \neq 1$ for some $g \in G$, then $c \in H^{2}$ (since $G / H^{2}$ is Abelian). This completes the proof that $G$ is a $Q^{*}$-group. Moreover, if $a \in H$ satisfies $a^{2}=c$, then $\left[z_{2} a^{-1}, g\right]=1$ for all $g \in G$ and so $z_{2} \in\langle a\rangle Z_{1}(V(\mathbb{Z} G))$. Since the subgroup $H$ is uniquely determined in a non-Dedekind $Q^{*}$-group, we conclude that $Z_{2}(V(\mathbb{Z} G)) \subseteq\langle a\rangle Z_{1}(V(\mathbb{Z} G))$ and this section of the proof is complete.

Finally, assume that $G$ is Dedekind. Recall that $z_{2} \in Z_{2}(V(\mathbb{Z} G)) \backslash Z_{1}(V(\mathbb{Z} G))$. Hence $G$ is not Abelian, so $G \cong Q_{8} \times E_{2} \times E_{2}^{1}$ where $E_{2}$ is an elementary Abelian 2-group and $E_{2}^{1}$ is an Abelian group in which every element is of odd order.

We claim that $E_{2}^{1}=\{1\}$. Say this is not the case and $x \in E_{2}^{1}$ is of order $p$ where $p$ is an odd prime. Note that $x$ is central in $G$. Since $z_{2}$ is not central, there must exist an element $y$ of order 4 in $Q_{8}$ such that $\left[z_{2}, y\right] \neq 1$. Since $\langle y\rangle \triangleleft G$ and $z_{2} \in N_{V(\mathbb{Z} G)}(G)$, we can show as before that $z_{2} y z_{2}^{-1}=y^{3}$. It follows that $x y$ is of order $4 p$ and $z_{2}(x y) z_{2}^{-1}=x y^{3}=(x y)^{2 p+1}$. As before, we now obtain a 
contradiction by examining either a suitable Bass cyclic (if $p>3$ ) or Hoechsmann (if $p=3$ ) unit in $V(\mathbb{Z} G)$.

Now we have $G \cong Q_{8} \times E_{2}$, a Hamiltonian 2-group and also a $Q^{*}$-group. In this case, Higman's theorem says that $Z_{2}(V(\mathbb{Z} G))=Z_{2}(G)=G$, as desired.

The proof is complete.

An immediate consequence of Theorem 2 is the result stated in the introduction.

Corollary 3. If $G$ is a torsion group, then $Z_{2}(V(\mathbb{Z} G))=T \cdot Z_{1}(V(\mathbb{Z} G))$ where $T$ is the torsion subgroup of $Z_{2}(V(\mathbb{Z} G))$.

\section{REFERENCES}

[1] Satya R. Arora, A.W. Hales and I.B.S. Passi, Jordan decomposition and hypercentral units in integral group rings, Commun. in Alg. 21 (1993), 25-35. MR 93m:20005

[2] Satya R. Arora and I.B.S. Passi, Central height of the unit group of an integral group ring, Commun. in Alg. 21 (1993), 3673-3683. MR 95a:20004

[3] Norman Blackburn, Finite groups in which the nonnormal subgroups have nontrivial intersection, J. Algebra 3 (1966), 30-37. MR 32:7643

[4] A.A. Bovdi, The periodic normal divisors of the multiplicative group of a group ring II, Sibirsk. Mat. Z. 11 (1970), 492-511. MR 43:4930

[5] Yuanlin Li, The hypercentre and the $n$-centre of the unit group of an integral group ring, Canad. J. Math. 50 (1998), 401-411. MR 99f:16034

[6] Yuanlin Li, M.M. Parmenter and S.K. Sehgal, On the normalizer property for integral group rings, Commun. in Alg. 27 (1999), 4217-4223. CMP 99:17

[7] M.M. Parmenter, Conjugates of units in integral group rings, Commun. in Alg. 23 (1995), 5503-5507. MR 96m:16047

[8] M.M. Parmenter, Central units in integral group rings, Algebra: Some Recent Advances, INSA, 111-116, 1999. MR 2000d:16047

[9] S.K. Sehgal, Topics in Group Rings, Marcel Dekker, 1978. MR 80j:16001

[10] S.K. Sehgal, Units in Integral Group Rings, Longman, 1993. MR 94m:16039

Department of Mathematics and Statistics, Memorial University of Newfoundland, St. John's, New Foundland, Canada A1C 5S7

Department of Mathematics and Statistics, Memorial University of Newfoundland, St. John's, New Foundland, Canada A1C 5S7 